ELOHI

Peuples indigènes et environnement

$8 \mid 2015$

Exodes, déplacements, déracinements

\title{
Peuples et territoires indigènes dans les romans de Milton Hatoum : une écriture de l'oubli
}

Mireille Garcia

\section{(2) OpenEdition}

Journals

Édition électronique

URL : http://journals.openedition.org/elohi/893

DOI : 10.4000/elohi.893

ISSN : 2268-5243

Éditeur

Presses universitaires de Bordeaux

Édition imprimée

Date de publication : 1 juillet 2015

ISBN : 979-10-300-0130-3

ISSN : $2437-8175$

\section{Référence électronique}

Mireille Garcia, «Peuples et territoires indigènes

dans les romans de Milton Hatoum : une écriture de l'oubli », ELOHI [En ligne], 8 | 2015, mis en ligne le

01 juillet 2015, consulté le 07 mai 2019. URL : http://journals.openedition.org/elohi/893; DOI :

10.4000/elohi.893 


\title{
Peuples et territoires indigènes dans les romans de Milton Hatoum: une écriture de l'oubli
}

\author{
MIREILLE GARCIA \\ ERIMIT, Université Rennes II
}

Qu'ils soient de nature littéraire ou encore des textes d'information, les écrits des voyageurs et explorateurs européens ont largement contribué à la création d'un imaginaire de la nation privilégiant une image du Nouveau Monde qui oscille entre le mystique et le fantastique, l'exotique et le pittoresque, le mystérieux et l'inconnu. En effet, le thème de l'occupation du Brésil et du sort des Amérindiens depuis les premiers temps de la conquête est aussi celui de l'appropriation d'un espace imaginé, considéré comme vierge.

C'est probablement à la région de l'Amazonie et à sa population principalement indigène que le concept « d'histoire inventée » convient le mieux car il fait référence à un passé historique considéré comme inexistant, mais représente aussi et surtout une réflexion autour des possibilités de construction et d'institutionnalisation des traditions. Dans son étude intitulée Breve história da Amazônia, Márcio Souza parle "d'histoire inventée », concept qui selon lui attribue à l'Amazonie un passé historique imaginaire, symbolique et fragmenté, et l'a transformée en un territoire sans tradition culturelle ou histoire:

Com a onda de cientistas viajantes, começa a ser fabricado o renitente mito de que a Amazônia é um vazio demográfico, uma natureza hostil aos homens civilizados, habitada por nativos extremamente primitivos, sem vida política ou cultural. É a Amazônia terra sem história, que tem permitido toda sorte de intromissão e arbitrariedade. (Souza 76).

Selon lui, l'Amazonie a été "inventée » par les colonisateurs qui pensaient construire une unité mais n'ont fait quaffirmer les différences et démarquer les frontières avec le reste de la nation. Cette modification de perception de 
l'Amazonie est due aussi aux voyageurs et explorateurs qui, par le biais de leurs récits, ont décrit une région à la fois fantastique, méconnue et mystérieuse, où les populations autochtones deviennent l'objet du colonialisme :

As narrativas dos primeiros viajantes [...] como representação - quer fossem uma lição ou necessidade - ofereciam ao mundo uma nova cosmogonia : dramaturgia de novas vidas ou espelho de novas possibilidades, tal era o espírito de todas elas, enunciando e formulando o direito de conquistar dos desbravadores europeus. (Souza 34).

Ces chroniques ont favorisé la déconstruction de l'univers multiculturel amazonien : transformée en une région cataloguée, interprétée et classée au détriment de son historicité, l'Amazonie se voit " séquestrée » au profit du projet colonial - ainsi que l'altérité de l'Indien qui l'habite - ce qui provoque une véritable collision culturelle, raciale et sociale. Dès lors, « l'histoire de la colonisation s'ajoute à celle de l'Amazonie, antérieure et méconnue, de peuples dont l'histoire fut niée, réduite au néant par une remise à zéro qui fixa les origines à l'instant des Découvertes " (Thiérion 55). D’après Márcio Souza, la révélation de l'Amazonie marque le début du phénomène de dépersonnalisation des populations indiennes et le refus de reconnaître leur altérité. L’effacement identitaire de l'indigène se fait peu à peu : véritable processus de " détribalisation » comme il le nomme, ces mouvements de mutations et de transformations des populations contraintes à un métissage forcé vont donner naissance à toutes sortes d'appellations diverses résultant du processus historique de l'Amazonie et de son développement : l'Amazonéité ou encore les Amazonides sont autant de termes qui identifient les êtres déculturés de la région de l'Amazonie. L'émergence de l'identité cabocla en est l'exemple le plus notoire : élément incontournable de l'Amazonie, le caboclo en est la figure prédominante. Márcio Souza explique que le phénomène de " caboquização » des Indiens est apparu à la période d'indépendance du Brésil ; ces indigènes acculturés par leur contact avec les colonisateurs, issus de cultures différentes et réunis de manière stratégique afin d'en faire un réservoir de main d’ouvre esclave, sont le fruit de la colonisation portugaise, résultat de la miscégénation ${ }^{1}$.

1 À ce sujet, voir la thèse de doctorat de Brigitte Thiérion dans laquelle son auteure explique que «le caboclo est le produit de multiples croisements interethniques résultant de la politique de miscigénation encouragée par la couronne portugaise auprès des populations pauvres [...]. À l'origine le caboclo est dénommé Tapuio (métis d'Indien et de Blanc), il est un paria dans la société, l'appellation comporte une nuance dépréciative. Il se situe dans un entre-deux, car il nest plus un Indien, bien qu'il lui soit apparenté, et il ne sera jamais intégré à la société coloniale qui ne cherche quà l'asservir ou à l'utiliser par diverses mesures coercitives [...] Comme résultat d'un processus de peuplement, il est vu comme une mutation, tantôt interprété comme une régression 
On parlera alors d'Indiens génériques, détribalisés, abâtardis et dégénérés; de sujets transplantés, amputés, privés d’origine et d'histoire, oubliés; de communautés culturellement syncrétiques; de transfiguration ethnique; en somme, d'une identité insaisissable où la notion de perte engendre un sentiment de honte et de marginalisation.

À l'instar de la littérature de voyage qui a ainsi exploité et façonné les images de cette Amazonie « oubliée » de tous, lécrivain Milton Hatoum a lui aussi posé un nouveau regard sur l'Amazonie. Selon lui, elle se définit comme une entité construite dans l'imaginaire, comme territoire fragmenté qui « n’a pas de frontières mais des délimitations de frontières ${ }^{2}$ » même si elles ne sont qu'imaginaires. Il ajoute que « pour toutes les personnes nées en Amazonie, la notion de terre sans frontières et très présente et significative car il s'agit d'un univers très vaste » (Hatoum 1993b). Par ailleurs, ses romans mettent en scène des représentants des populations indigènes - marginalisées, stigmatisées et désappropriées - qui tentent tant bien que mal de lutter pour la sauvegarde de leurs valeurs, de leurs traditions et de leur territoire. Sa diégèse repose en effet sur la valorisation des contributions indigènes dans la construction d'une identité brésilienne, et pose en particulier la question de l'intégration des Indiens à une société qui les marginalise.

C'est pourquoi dans le présent article notre propos sera d'indiquer de quelle manière les peuples et territoires indigènes sont représentés dans les romans de Milton Hatoum Récit d'un certain Orient, Deux frères et Cendres d'Amazonie : Il s'agira de montrer quelle place les Amérindiens occupent sur le vaste territoire amazonien dépeint par l'auteur et par là même de comprendre comment cette place permet de discuter les relations entre territoire, identité et mémoire.

\section{Les indigènes de Milton Hatoum : marginalisation et « détribalisation »}

La représentation de l'Amazonie passe incontestablement par la représentation de sa population amérindienne, c'est pourquoi les récits de Milton Hatoum mettent en scène des personnages d'ascendance indigène. Pour la plupart « dé-

par rapport à l'identité amérindienne pure, tantôt comme une alternative heureuse, une survivance des peuples amérindiens [...] une ethnie dotée d'une culture résiduelle indigène " (Thiérion 155).

2 «A Amazônia não tem fronteiras; sim há uma delimitação de "fronteiras", mas para nós não passam de fronteiras imaginárias. Que importa, para os indio yanomamis, por exemplo, se eles foram assassinados na Venezuela ou no lado brasileiro? Para os índios, o território, a terra deles não tem fronteiras... "(Hatoum 1993b). L'auteur entend par là que l'Amazonie ne possède pas de frontières aux yeux des populations amérindiennes. 
tribalisés » et acculturés, tantôt animalisés, tantôt décrits comme des êtres assujettis, ces « Amazonides » déculturés ont perdu leur identité et sont devenus des étrangers sur leur propre territoire. Intégrés à des classes inférieures d'extrême pauvreté de la société brésilienne, ces Indiens sont relégués aux marges de la nation. Il s'agit ici de la condition de «estrangeiro de dentro» - expression que Rita Olivieri Godet emploie afin de rendre compte de la réalité des populations indigènes dans le contexte actuel de nos sociétés - qui témoigne de la place qu'occupent ces "étrangers » dans le processus de construction des identités plurielles et complexes des Amériques dans un contexte où les imaginaires archaïques cohabitent aux côtés des imaginaires planétaires. Selon elle, «le questionnement de la figuration de l'amérindien comme "estranho estrangeiro de dentro" nous aide à comprendre la place que les sociétés urbaines réservent à ces populations " (Olivieri-Godet 2009 : 91).

Dans les trois romans dont il est ici question, une multitude de personnages est symbolique de la représentation de la société amérindienne marginalisée. Parmi eux, les caboclos- en majorité reconvertis en vendeurs ambulants - errent aux quatre coins de la ville et les caboclas - devenues filles de joie - emplissent les maisons closes. Les indigènes apparaissent comme étant les stigmates de la société, marginalisés et réduits à la misère, également victimes de fortes discriminations comme en témoignent les passages suivants : « La colonne de mendiants et de gamins misérables grossissait d’année en année [...] qui venaient montrer leurs plaies et leurs membres estropiés " (Hatoum 1993a : 122-123); " Assis sur les marches de l'église, des Indiens et des déracinés venus de l'intérieur mendiaient » (Hatoum 2003 : 240) ; « Une famille d'Indiens ramassait les pièces de monnaie qu'il (Mundo) avait lancées; ils habitaient là, entre la grille et la façade de la maison en ruines » (Hatoum 2008 : 41) ; " Avant, ces Indiens étaient soignés par des guérisseurs, des escrocs du corps et de l'âme. Nous payons le docteur Kazuma, pourtant cela ne les empêche pas de continuer à être des sauvages, des ingrats. Ils oublient nos efforts, notre dévouement. Ils sont comme des enfants... » (Hatoum 2008 : 78).

Ces propos stigmatisants et brutaux - dans lesquels la référence au colonialisme est explicite - dénotent une vision eurocentrique basée sur les préjugés des colonisateurs qui voyaient dans les populations autochtones une foule de sauvages anonymes sans identité apparente qu'il fallait dompter et maitriser, exprimant ainsi leur rejet et leur infériorité ; attitude qui, au demeurant, révèle que la vision colonialiste perdure. Par conséquent, ces personnages secondaires renforcent le sentiment d'étrangeté que suscite la culture indigène aux yeux des autres habitants de la région, et se voient attribuer un statut d’étrangers sur leur propre territoire. 
Toutefois, parmi ces sujets épisodiques - et fantomatiques - qui peuplent les romans, nous retrouvons systématiquement un ou deux personnages emblématiques par le biais desquels l'auteur nous offre la possibilité d'entendre la voix des opprimés, des oubliés, des Amazoniens natifs.

Dans Récit d'un certain Orient, l'Indien et guérisseur Lobato Naturidade apparaît comme le détenteur de la culture amérindienne et de ses mystères : surnommé « le "Prince de la Magie Blanche" [...] il parlait couramment le nheengatu [...] À son arrivée à Manaus, on ne l'appelait que Tacumã, son vrai nom, et il était considéré comme un voyant» (Hatoum 1993a : 113-114). Bien que fictif, le personnage porte le même nom que le leader spirituel des Kamayu$\mathrm{ra}-$ une des quatorze tribus indigènes qui vivent au sud du Parc National du Xingu - en lutte pour la sauvegarde de leur langue et culture. Peut-être est-il possible d'associer le personnage fictif au personnage réel, même si l'auteur ne le revendique pas.

Dans le roman Deux frères, c'est certainement le personnage de Domingas ${ }^{3}$ qui est le plus représentatif de la problématique identitaire des indigènes. Cette cunhantã orpheline recueillie par la famille protagoniste du roman incarne la " détribalisation » : baptisée et alphabétisée, elle est l'avatar de l'Indien acculturé, elle symbolise la fabrication d'un individu nouveau en ce qui concerne la religion et les lettres jusquà être privée de son nom d'origine au profit d'un nom d'emprunt - ou de baptême - associé à la religion catholique et à ses rites dominicaux. Toutefois, au personnage soumis et tributaire qu'elle représente vient se greffer une autre facette, celle de l'Indienne récalcitrante vivant un conflit intérieur, une double appartenance, à l'image de toute la société indigène acculturée :

Domingas, la petite Indienne maigrichonne, esclave et nourrice à la fois, qui n’avait pour sa part d’autre idée que dêtre libre. Elle me l’a dit un jour, épuisée, nen pouvant plus, enchainée au destin de cette famille, comme les autres servantes du voisinage, elles aussi alphabétisées et éduquées par les religieuses des missions. (Hatoum $2003: 65$ ).

Tout comme le personnage de Lobato Naturidade, c'est au moyen de son savoir qu'elle tente d'assurer la survie de sa culture : elle sérige en guérisseuse, prodiguant des soins à partir des connaissances qu'elle avait tirées des plantes

3 À ce sujet, voir l'entrevue dans laquelle Milton Hatoum révèle son processus de création du personnage de Domingas influencé par Gustave Flaubert (Hatoum 2004). Il en ressort que ce personnage flaubertien incarne l'esclavage dissimulé de ces êtres sacrifiés à la fois par la société et par le destin, dont le dévouement ne connait pas de bornes car ils sont confrontés, comme le déclare l'auteur, à ce « manque de choix » que leur impose l'esclavage. 
médicinales de l'Amazonie, et elle détient les secrets des mystérieuses légendes et des mythes propres à la culture amérindienne.

Enfin, et contrairement aux deux œuvres précédentes, dans Cendres d'Amazonie, c'est un des principaux protagonistes qui sérige en grand défenseur de la cause indigène; fils d'un riche héritier d'origine portugaise et d'une mère d'ascendance amérindienne, le jeune artiste Mundo déclare sans détours que sa mère et lui sont "de quelque tribu oubliée " (Hatoum $2008: 16$ ) et revendique ses origines par le biais de ses œuvres d'art en créant une série de toiles à l'effigie de la culture indigène : « il avait d'abord dessiné sur une toile une carte de l'Amazonie $[\ldots]$ et il avait inscrit de nombreux noms indigènes, avec une croix à côté de chaque tribu " (Hatoum 2008 : 236-237). Mais c'est aussi sous l'influence de l'artiste Arana que Mundo va s'investir dans ses créations. En effet, Alduíno Arana, le "visionnaire de l'Amazonie " se consacre lui aussi à une œuvre qui reflète la réalité indigène et qu'il intitule «La Douleur des tribus... La Douleur de toutes les tribus» (Hatoum 2008 : 115) et dont le nom dénonce la disparition des populations indigènes : « ses toiles représentaient des paysages peuplés de caboclos et d'Indiennes nues à la peau cuivrée et au sourire complaisant " (Hatoum 2008 : 234). Son combat pour la reconnaissance de la cause indigène se reflète dans cette œuvre qui ne décrit pas seulement l'Amazonie pittoresque mais aussi l'anéantissement et l'abandon des tribus comme il en témoigne en expliquant la visée de sa toile : «Des dépouilles de notre peuple... d'Indiens et de caboclos [...] La nature, à elle seule, ne sert pas à grand-chose. Les ossements dêtres anonymes sont plus qu'un symbole " (Hatoum 2008 : 114). Par conséquent, il est concevable que ces œuvres d'art puissent représenter la recherche d'un langage capable de traduire la réalité de l'Amérindien.

Cependant, et malgré la volonté de l'auteur de donner de la voix à ces personnages contestataires qui dénoncent la triste réalité des populations indigènes ou répandent autour d'eux des éléments qui contribuent à la préservation de leur identité, force est de constater qu'ils sont tout de même la cible de propos diffamatoires critiquant leurs traditions et leurs cultes. Les pratiques religieuses de ces populations indigènes sont l'exemple notoire de la ségrégation dont sont victimes les personnages amérindiens de Hatoum, à l'instar du guérisseur Lobato Naturidade de Récit d'un certain Orient qui se voit relégué au rang de sorcier profane aux méthodes jugées peu orthodoxes :

On racontait, de bouche-à-oreille, que le guérisseur avait déjà empoisonné et aveuglé de pauvres diables $[\ldots]$ on parlait aussi de rituels diaboliques qui attiraient l'esprit du Mal pour le faire entrer dans les entrailles de la victime. Il s'en trouva encore pour vilipender ses méthodes [...] Ces traits infâmes et les anathèmes lancés contre un homme paisible qui ne se faisaient jamais remarquer étaient autant de traits 
décochés contre une tradition encore vivante qui palpitait au cœur des quartiers périphériques. (Hatoum 1993a : 117-118).

Le roman Deux frères dénonce lui aussi les préjugés envers les Indiennes païennes, «ces Indiennes qui étaient capables de tout avec les enfants, on parlait de cas d'étranglement, de vampirisme, d'empoisonnement, ou de crimes encore pires » (Hatoum $2003: 66$ ).

Quant au récit Cendres d'Amazonie, on y retrouve une critique ouverte de la société envers les rituels syncrétiques mi-indigènes et mi-chrétiens pratiqués par les Amérindiens : « un jour ils prient Notre-Dame du Carmel, le lendemain ils oublient la sainte et l'Église. La foi de ces gens-là est comme une girouette " (Hatoum 2008 : 79).

Aussi, on comprend que les pratiques et les croyances indigènes sont une incompréhension totale et une énigme indéchiffrable pour les élites qui relèguent leurs adeptes au rang d'impies mécréants et profanes.

C'est à partir de telles considérations que nous interrogeons la place que tiennent les populations indigènes sur le vaste territoire amazonien dès lors qu'il apparaît que leur intégration y est difficile, voire impossible. Occupant une position marginale et illégitime, les indigènes s'apparentent à de véritables orphelins possédant un statut ambivalent voire indicible, statut qui, selon nous, permet d'éclairer les processus de construction et de déconstruction au sein de la société amazonienne. En effet, ces " orphelins de la civilisation ", comme les appelle Rita Olivieri-Godet, sont la métaphore de la «souffrance d'un peuple appauvri par la politique gouvernementale [...] un peuple orphelin, abandonné, marqué par un sentiment de tragique impuissance " (Olivieri-Godet 2009 : 101-107). Par conséquent, ces indigènes orphelins et en marge ne se constituent pas uniquement comme des êtres solitaires : ils s'insèrent dans une grande métaphore de l'abandon des populations amérindiennes de l'Amazonie et caractérisent la réalité sociologique et géographique de la fragmentation et de la « non-appartenance ». Afin d'approfondir cette réflexion, il importe à présent d'analyser l'espace symbolique indigène que dépeint le romancier dans ses œuvres, ou plutôt les lieux symboliques de l'oubli que représentent ces espaces au même titre que leurs occupants.

\section{Terres indigènes : espaces du dehors et de l'oubli}

Les terres amazoniennes s'avèrent être un lieu devenu insaisissable, aux frontières floues et indéfinies, comme en témoigne l'auteur lui-même qui déclare se trouver face à l'impossibilité de décrire et de situer l'Amazonie, cet « espace blanc $[\ldots]$ endroit lointain, territoire perdu dans les tréfonds de la forêt et dans un recoin obscur de l'histoire» (Hatoum 1993b). Par ailleurs, ses romans s'ins- 
crivent dans la perspective historique de la ville de Manaus où d'importantes transformations s'opèrent : phase de développement et de modernisation de la ville - et par là même de l'Amazonie tout entière - de la «Belle-époque tropicale ", époque de faste du cycle du caoutchouc et de l'expansion du capitalisme, durant laquelle les élites dirigeantes cherchaient le luxe et l'ostentation dans l'architecture et l'urbanisme, s'efforçant de véhiculer une image exemplaire de la région et de l'État Amazonien. Dès lors, la représentation d'une contrée opulente, fertile et imposante - qui se devait dêtre à l'image du reste de la Nation - s'imposait, mais ne pouvait se produire qu'en dépit des populations indigènes : susceptibles d'enrayer l'évolution et le processus de "civilisation" de l'Amazonas et de ternir son image, elles ont été occultées, voire entièrement effacées et désappropriées de leurs terres. À ce propos, la récente étude de Rita Olivieri-Godet examine les processus de "civilisation " qui exterminent la «barbarie » afin de faire naître une nouvelle nation :

Num romance que situa a ação no século XIX, período de polarizações extremas na história do Brasil, no âmbito internacional e no interior do estado nacional em formação, questiona-se o modelo que direcionou esse processo, baseado na ideologia do progresso, do desenvolvimento e da civilização, ideais que orientam a política de extermínio e de assimilação das nações indígenas.(Olivieri-Godet 2013 : 140).

Bien que ce commentaire ne concerne pas les romans de Milton Hatoum, il pourrait parfaitement s'y appliquer dans la mesure où ils mettent en scène la tentative de résistance des indigènes qui luttent tant bien que mal pour préserver leurs valeurs et leurs traditions. Malgré cela, on observe que la mémoire indigène dans la ville de Manaus (lieu de prédilection des romans de Hatoum) est quasi nulle, voire inexistante : aucune place, rue ou avenue narbore de nom indigène et aucune sculpture, statue ou monument n'est érigé en mémoire des Amérindiens. A ce propos, l'étude de Nascimento avance la thèse suivante :

Apresentamos uma recusa em nos identificarmos com os índios, nenhuma praça tem nome indígena, não há esculturas de índios ou mesmo do caboclo seringueiro decorando os logradouros públicos. Estas imagens estão dispostas em museus e casas de artesanato onde a maioria da população que visita, é estrangeira. Neste sentido, as obras colocadas pelo poder público refletem a imposição da memória oficial sobre a memória coletiva e individual, o que acabou por gerar esta nossa memória subterrânea [...] E o patrimônio construído na área do Centro Histórico reflete esse processo de modernização que foi vivenciado por muitas cidades em vários países, na passagem do século XIX para o século XX.(Nascimento 41).

Ainsi, il paraît évident que les notions de trace et de vestige s'appliquent à cette mémoire indigène réprimée que l’on a tenté d’effacer : comme enfouies 
et dissimulées, les traditions indigènes ne peuvent être remémorées qu'à travers loubli, et ceci est visible en particulier dans certains lieux de Manaus qui recèlent de véritables reliques ensevelies ayant appartenu à diverses populations amérindiennes. Duarte ainsi que bon nombre d'historiens s'accordent à dire que Manaus est à l'origine une immense crypte sur laquelle la ville s'est bâtie :

Manaus é considerada pelos estudiosos no assunto como um grande cemitério indígena em razão dos mais de cinquenta sítios arqueológicos urbanos aqui existentes. Ao longo da história, a área onde tem ocorrido o maior número de descobertas de urnas funerárias indígenas localiza-se no espaço compreendido pelas praças Nove de Novembro e Dom Pedro II, no Centro.(Duarte 147).

Ses propos sont très proches de ceux de Nascimento qui parle de ces vestiges comme étant le fruit d'une "mémoire souterraine». Elle explique qu'aujourd'hui la ville de Manaus se trouve "confrontée à l'immersion [...] déléments qui ramènent à la surface la mémoire souterraine. Il s’agit d’objets artisanaux qui ont été découverts, révélant une ville souterraine dont l'histoire et la mémoire diffèrent de celles de la ville construite » (Nascimento 35).

L’enfouissement des indigènes est brièvement évoqué dans les romans de Hatoum. Alors que l'un des narrateurs reconnaît que " le cimetière était plus vaste que la ville " (Hatoum 1993a : 92), le personnage d'Adamor Piedade — qui n'est autre que le fossoyeur du cimetière - revient sur ses années de service à inhumer inconsidérément les corps de centaines de natifs démunis : " une vie toute entière à creuser la terre pour mettre les morts à l'abri ; il mentionna les défunts célèbres de la ville, et des va-nu-pieds enterrés au hasard, sans chérubins ni chants célestes, et sans illusions sur leur vie future" (Hatoum 1993a : 193). Et dans l'œuvre Cendres d'Amazonie, l'artiste Mundo révèle avoir dessiné, dans certaines de ses toiles « des Indiens et des caboclos [...] peints en arrière-plan [...] sur l'arrière-plan caché et honteux de notre histoire » (Hatoum $2008: 315$ ).

Bien que les allusions à une " mémoire souterraine " s'arrêtent à ces quelques exemples, les récits ressuscitent malgré tout les traditions indigènes et leur confèrent des lieux de survivance, à l'instar de la forêt amazonienne. Or, et bien qu'il y ait là une volonté de la part de l'auteur de nous figurer l'espace de la forêt de façon positive - à savoir tel le berceau de la civilisation amérindienne - il n'empêche que la sauvagerie et l'hostilité qui s'y rattachent dans l'imaginaire de la population l'emportent. La forêt renferme «des épidémies foudroyantes, des cruautés raffinées, des adorateurs de la lune, d'innombrables batailles aux couleurs crépusculaires, des hommes qui se régalaient de la chair de leurs semblables » (Hatoum 1993a : 87). Tout ce qui a trait à la forêt est comme dissimulé ou voilé, les Indiens vendant leur artisanat étaient «terrés au fond de leurs baraques [...] abîmés par le temps et par la négligence (leurs) visages [...] 
semblaient appartenir à ces êtres indifférents à tout" (Hatoum 1993a: 154) et la jungle elle-même est occultée : " de gros cargos amarrés dans Manaus Harbour masquaient l'horizon de la forêt " (Hatoum 2003 : 240). En somme, cet univers auquel les traditions indigènes appartiennent représente " l'insaisissable (qui) ne peut être reproduit mais doit être recréé » (Hatoum 1993a : 156).

Outre la forêt, de nombreuses localités au patrimoine et aux noms indigènes sont brièvement citées dans les récits sans jamais être décrites en détail. Il s'agit, pour la plupart, d’endroits reculés ou difficiles d'accès, très peu ou mal connus et relégués au second plan.

Si la diégèse de Récit d'un certain Orient se cantonne principalement au centre de Manaus, les deux autres romans vont au-delà des frontières de la ville. Dans Deux frères, la domestique Domingas cherche à retrouver son village natal, endroit lointain et retiré de la ville de Manaus, lieu dénonciation de la trame : «le lieu où elle était née, près du village de São João, sur la rive du Jurubaxi, un bras du Negro, très loin d'ici » (Hatoum 2003 : 72). Puis, lorsque le personnage d'Omar disparait, sa famille se met à sa recherche en écumant les endroits les plus lointains et perdus :

Ils allaient fouiller tous les lacs et toutes les rivières dans leurs moindres recoins [...] Pendant des semaines nous avons navigué en faisant de grandes boucles. Nous sortions très tôt le matin, contournions l'île Marapata, traversions le Xiborena jusquà l'île de Marchanteria. Puis, engagés dans le Solimões, nous entrions dans le paraná du Careiro, et tracions un arc de cercle jusquà l’Amazone [...] Nous avons longé tous les rivages de la Terra Nova, du Marimba, du Murumurutuba... Nous avons fait le tour de tous les lacs sur l'île du Careiro : le Joanico, le Parun, l'Alencorne, l'Imanha, le Marinho, l'Acara, le Pagao [...] les berges du Cambixe [...] Humaïta [...] Itacoatiara [...] Mais des îles, il y en avait des centaines. (Hatoum $2003: 161-163)$.

À première vue, lénumération de tous ces toponymes ne semble pas pouvoir nous apporter quelque précision que ce soit sur la question. Toutefois, cette démarche énonciative n'est pas sans rappeler la dynamique rotatoire du cercle qui révèle au lecteur des espaces entremêlés, comme enchevêtrés. En effet, la répétition de ces lieux et leur énumération "en boucle " traduit la volonté d'assoir leur authenticité et, par conséquent, de les ancrer dans la mémoire. En outre, il est intéressant de noter que ces espaces, encerclés par les eaux, sont en majorité des îles dont la signification symbolique nous renvoie au paradis originel, à l'autre-monde et à l'au-delà merveilleux. Nettement délimitée et presque préservée de la civilisation, l'île est le lieu utopique par excellence ; elle incarne toutes les aspirations de l'imaginaire et illustre parfaitement le mythe 
de la terre-mère et du lieu de refuge ${ }^{4}$. Notons également que la notion d'engloutissement et de submersion se rattachent aussi à l'insularité.

Ainsi, l'aspect insulaire de ces localités dit à la fois leur éloignement et leur inaccessibilité comme leur caractère empreint $d^{\text {' }}$ "ailleurisme ${ }^{5} »$ : elles deviennent l'endroit de l'Autre, l'espace du dehors, comme par exemple dans Cendres d'Amazonie où le narrateur va découvrir des lieux tels que Saracura, Arari et Itaborai ${ }^{6}$ où des vestiges des caboclos subsistent (Hatoum 2008 : 75-76).

Nous pouvons donner d'autres exemples dans ce même récit où la représentation de ces espaces est également elliptique et brève : lorsque le personnage de Ranulfo disparaît - dans un besoin de fuir le domicile conjugal - il s'introduit presque subrepticement des villes distantes de Manaus : il « voyageait dans la région de Santarém, Oriximiná, Óbidos et Monte Alegre ; il irait jusqu’à Belém, puis reviendrait à Manaus » (Hatoum 2008 : 62). Le yacht qui conduit Mundo et son père à la Vila Amazônia où ils possèdent une résidence secondaire fait d'abord une escale à Urucurituba, petit village où « des chemins de terre finissaient dans la forêt » (Hatoum $2008: 67$ ) et où ils troquent des denrées alimentaires contre des fruits, du poisson ou des tortues avec des caboclos vivant dans cet endroit qui semble coupé du reste du monde. Puis ils naviguent ensuite sur le Rebojal, "zone où les eaux sont rudes » et où " un brouillard visqueux recouvre les rives et la forêt, empêchant de distinguer l'horizon » (Hatoum 2008 : 69). Enfin, ils font escale à Urucará où « une pluie épaisse cacha la forêt et l'horizon» (Hatoum $2008: 72$ ). Il est intéressant de noter ici à quel point le climat peut avoir une incidence sur ces espaces qui semblent s'effacer, s'estomper pour enfin disparaitre totalement.

4 À ce propos, il est intéressant de citer les travaux de Rita Olivieri-Godet concernant le "paradoxe de l'insularité »: " L'espace circonscrit de l'île fonctionne comme une métaphore du pays dans sa totalité. L'île figure de manière emphatique la limitation de frontières spatiales et culturelles de tout territoire qui se constitue comme nation, avec un peuple, une langue et une culture qui s'affirment comme distincts des autres. $\mathrm{La}$ figuration d'une image insulaire du pays renvoie à ses origines historiques, explorant les tensions entre images réelles et utopiques de l'île Brésil, tissées à travers le temps » (Olivieri-Godet $2005:$ 49-50).

5 Nous reprenons ici le néologisme employé par Zilá Bernd (Bernd 1995).

6 Il est important de préciser que lédition française du roman ne retranscrit pas fidèlement certains toponymes d'origine indigène cités dans la version originale. C'est ici le cas pour cette localité d'Itaboraí où le terme apparaît sans accent graphique dans la version française. Il peut s'agir d'un oubli du traducteur ou bien de la volonté d’adapter le mot à lorthographe française. Dans tous les cas, aucune note de traduction ne justifie un tel choix. 
Un point de discussion est possible autour de l'origine des noms à consonance indigène de tous ces endroits, ce qui expliquerait que l'auteur ait voulu rendre compte du caractère insaisissable, mystérieux, caché et secret des traditions indigènes qui ne subsistent que par la trace ou encore le vestige. En se soustrayant à la description de ces lieux, le romancier dit en quoi le silence et l'oubli s'y reflètent, leur attribuant presque un air de lieu inexistant d'indistinction et d'omission. Paradoxalement, ces espaces isolés en arrière-plan, aux allures de territoires déshumanisés et vidés de leurs histoires, semblent être les seuls susceptibles de protéger les hommes de la dépersonnalisation du monde moderne urbain. Voilà pourquoi il semble pertinent d'associer ces lieux à un espace mystérieux voire surnaturel, empreint d'une certaine magie, qui pourrait être une fuite du monde réel. Les notions de terre imaginaire et d'histoire inventée du territoire amazonien prennent alors ici tout leur sens.

En définitive, en nous interrogeant sur la représentation des populations indigènes et à la place qui leur est conférée sur le territoire amazonien dans l’œuvre de Milton Hatoum, il nous a été possible de constater que les espaces - privés de contours définis - sétendent à un " ailleurs ", symbole de déterritorialisation et de déracinement. Les déplacements et les mouvances des personnages soulignent, par ailleurs, le caractère frontalier et indéfini du territoire amazonien au même titre que la reconstitution de leurs itinéraires dans des espaces inextricables et dédaléens témoigne de leur propre existence d' « orphelins de la civilisation ", dépossédés et condamnés à l'errance.

Si l'on examine la littérature actuelle (c'est-à-dire environ de 1980 à nos jours) produite dans les Amériques, on perçoit un imaginaire hanté par des représentations antagoniques: D’un côté, ce sentiment est révélateur de la souffrance et du rejet éprouvés par des peuples autochtones qui se sont vus dépossédés de leur culture et de leur territoire, somme toute, de leur identité. De l'autre côté, cet imaginaire abrite un sentiment de volonté de renaître et de tout recommencer. Les œuvres de Milton Hatoum s'insèrent dans la production romanesque actuelle et tout particulièrement dans cette optique de la mise en fiction des modulations sociales et territoriales liées à l'internationalisation de la région amazonienne. $\mathrm{Du}$ fait qu'ils posent la question de la place des populations Amérindiennes et de leurs territoires, ses romans abordent également un des problèmes actuels de société qui est le thème majeur de la condition des Amérindiens au Brésil. Ils proposent une réflexion sur l'exclusion de l'Indien du corps de la Nation, de tous les peuples relégués aux oubliettes de la construction de l'identité nationale. 


\section{Bibliographie}

BERND, Zilá. Littérature brésilienne et identité nationale : dispositifs d'exclusion de l'Autre. Paris : L'Harmattan, 1995.

DUARTE, Durango Martins. Manaus entre o passado e o presente. Manaus : Ed. Mídia Ponto Comm, 2009.

HATOUM, Milton. Cendres d'Amazonie (Traduit du portugais par Geneviève Leibrich). Paris, Actes Sud, 2008.

HATOUM, Milton. Récit d'un certain Orient (Traduit du brésilien par Claude Fages et GabrielIaculli). Paris : Editions du Seuil, 1993a.

HATOUM, Milton. Deux frères (Traduit du portugais par Cécile Tricoire). Paris : Seuil, 2003.

HATOUM, Milton. «Entrevista concedida a A. R. Hanania ». Revista Collatio $\mathrm{n}^{\circ}$ 6. 1993b. Disponible sur : http://www.hottopos.com/collat6/milton1.htm, consulté le 10 novembre 2014. 1993b

HATOUM, Milton. «Entrevista concedida a S. Vilela ». DW-WORLD. 2004. Disponible surhttp://www.dw.de/o-arquiteto-da-mem\%/C3\%B3ria/a-1355392, consulté le 20 janvier 2009.

NASCIMENTO, Maria. Patrimônio e memória da Cidade: Monumentos do Centro Histórico deManaus, dissertação de Mestrado, UFAM, 2003.Disponible sur www.slideshare.net/evanynascimento/patrimnio-e-memria-da-cidademonumentos-do-centro-histrico-de-manaus, consulté le 6 février 2014.

OLIVIERI-GODET, Rita. A alteridade ameríndia na ficcão contemporânea das Américas: Brasil,Argentina, Quebec. Belo Horizonte : Fino Traço, 2013.

OLIVIERI-GODET, Rita. "O ameríndio como personagem do Outro na literatura contemporânea : Órfãos do Eldorado e Nove noites ".Revista Brasileira de Literatura Comparada, $\mathrm{n}^{\circ} 15$ (2009), 89-111.

OLIVIERI-GODET, Rita. João Ubaldo Ribeiro. Littérature brésilienne et constructions identitaires, Rennes : PUR, 2005.

SOUZA, Márcio. Breve história da Amazônia. São Paulo : Marco Zero, 1994.

THIÉRION, Brigitte. Regards sur l'Amazonie: Fiction Histoire Identité dans l’ouvre de Márcio Souza, thèse de doctorat, Université Rennes 2, 2003.

\footnotetext{
Résumé : Parmi les productions littéraires contemporaines dont la diégèse repose sur la valorisation des contributions indigènes dans la construction d'une identité brésilienne, figurent les romans de Milton Hatoum Récit d'un certain Orient, Deux frères et Cendres d'Amazonie. Ces œuvres mettent en scène des représentants des populations indigènes - marginalisées, stigmatisées et désappropriées - qui luttent pour la sauvegarde de leurs valeurs, de leurs traditions et de leur territoire et posent la question de l'intégration des Indiens à une société qui les marginalise. Dans le présent article, notre propos sera d'indiquer de quelle manière les peuples et territoires indigènes sont représentés dans ces romans, de démontrer la place qu'occupent les Amérindiens sur ce vaste territoire amazonien et par là même de comprendre comment cette place permet de discuter les relations entre territoire, identité et mémoire.
} 
Mots-clefs : Milton Hatoum ; populations indigènes ; Amazonie ; territoire ; frontière ; déterritorialisation.

Mireille Garcia est titulaire d'un doctorat en littérature contemporaine brésilienne dont la thèse, soutenue en novembre 2014, porte sur la problématique de l'identité, de la mémoire et de l'espace dans l'œuvre du romancier Milton Hatoum. Prix de thèse 2015 attribué par l'Institut des Amériques. Membre de I'Equipe de Recherche Interlangue : Mémoires, Identités, Territoires (ERIMIT) et maitre de langue au Département de Portugais de I'Université Rennes 2.

Resumo : Dentre as produções literárias contemporâneas cuja trama trata da valorização das contribuições indígenas na construção de uma identidade brasileira, constam os romances de Milton Hatoum Relato de um certo Oriente, Doisirmãos e Cinzas do Norte. Estas obras colocam em cena representantes das populações indígenas - marginalizadas, estigmatizadas e desapropriadas - que lutam pela preservação de seus valores, de suas tradições e de seu território e questionam a integração dos Índios em uma sociedade que os marginaliza. No presente artigo, nosso propósito será de indicar a maneira com a qual os povos e territórios indígenas são representados nesses romances, de expôr o lugar que os Ameríndios ocupam nesse vasto território amazônico e assim compreender como este lugar permite discutir as relações entre território, identidade e memória.

Palavras-chave : Milton Hatoum, populações indígenas, Amazônia, território, fronteira, desterritorialização.

Mireille Garcia é titular de um doutorado em literatura brasileira contemporânea cuja tese, defendida em novembro de 2014, trata da problemática da identidade, da memória e do espaço na obra do romancista Milton Hatoum. Prêmio de tese 2015 atribuída pelo Institut des Amériques. Membro do grupo de pesquisa ERIMIT e professora substituta no Departamento de Português da Universidade Rennes 2. 